\title{
Mapping alpine snow using a spectral mixture modeling technique
}

\author{
Anne W. Nolin, Jeff Dozier and Leal A. K. Mertes \\ Center for Remote Sensing and Environmental Optics and Department of Geography, University of California at Santa Barbara, \\ Santa Barbara, California 93106, U.S.A.
}

\begin{abstract}
Remote sensing has provided a means of obtaining estimates of snow-covered area, yet traditional methods have had difficulty mapping snow in shaded and vegetated areas. Spectral mixture analysis is a linear mixture modeling technique that shows promise for mapping land surface covers, particularly when imaging spectrometer data are used. Applying this technique to AVIRIS data collected over the Sierra Nevada, California, we have estimated the fraction of snow cover in each pixel, even in areas that are shaded or forested. This modeling technique enables us to map snow cover at the sub-pixel level and provides a means of estimating the errors associated with the calculation.
\end{abstract}

\section{INTRODUCTION}

One of the fundamental difficulties in producing estimates of snow-covered areas using remote sensing techniques has been distinguishing snow from other surface covers in a scene. While snow is extremely bright in the visible wavelengths, it can also appear much darker when in the shadows or when it lies within a forested area, thus resulting in misclassification of snow-covered pixels. To date, methods for classifying snow-covered pixels from satellite data have relied on reflectance measurements in only a few bands and the use of thresholding and ratioing to distinguish between snow, clouds, and other scene components (Dozier and Marks, 1987; Dozier, 1989). There are several drawbacks to such approaches:

1. Removing atmospheric effects in mountainous terrain is difficult because of the changes in air mass with variation in elevation, leading to errors when calculating surface reflectance;

2. It is not possible to estimate snow-cover concentration at the sub-pixel level; pixels can only be classified as snow covered or not snow covered;

3. Typically, there is no means of performing an error assessment without extensive field sampling or analysis of aerial photos.

In this paper, we describe the application of a spectral mixture modeling technique for mapping snow-covered area and present results from two images of alpine regions in the Sierra Nevada, California.

\section{APPROACH}

With the advent of the age of imaging spectrometers, we are able to use both spatial and spectral data to extract information on a sub-pixel level (Goetz and others, 1985). The Airborne Visible and Infrared Imaging Spectrometer (AVIRIS), flown in NASA's ER-2 high altitude aircraft, has 224 spectral bands from 400 to $2460 \mathrm{~nm}$ and a pixel size of $20 \mathrm{~m}$. Since 1989, there have been three AVIRIS overflights over our region of interest, the Sierra Nevada of California, providing us with high spectral resolution images containing such components as snow, vegetation, rock, water, and shade. New techniques are evolving to interpret these data better and for this research we use a spectral mixture analysis method. Spectral mixture analysis has been shown to be well-suited for analysis of AVIRIS data (Boardman and Goetz, 1991; Clark and others, 1991; Sabol and others, 1992). In this context, spectral mixtures are areal composites (mixtures of components) within each pixel. While there are many components in a pixel, one can choose a parsimonious set of spectral endmembers that best describes the spectral variation in the pixel. A spectral endmember is the purest representation of a component in multispectral space. While an image component may itself be a mixture of sub-elements, it can be represented as a unique spectral signature. In this work, knowledge of the image allowed us to choose pixels that best represented the main components in the image. Having chosen these spectral endmembers, we can calculate the spectra of any linear mixture of the endmembers using the following mixing rule (Adams and others, 1986; Roberts and others, 1990):

$$
L_{\mathrm{c}}=\sum_{i=1}^{N} F_{i} L_{i, \mathrm{c}}+E_{\mathrm{c}}
$$

$L_{\mathrm{c}}$ is the radiometrically calibrated radiance in AVIRIS channel $\mathrm{c}\left(\mathrm{Wm}^{-2} \mu \mathrm{m}^{-1} \mathrm{sr}^{-1}\right) . F_{i}$ is the fraction of endmember $i . L_{i, \mathrm{c}}$ is the radiance of endmember $i$ in 
channel c. $N$ is the number of spectral endmembers. $E_{\mathrm{c}}$ is the error for channel c of the fit of $N$ spectral endmembers. The resulting fraction images show the relative contributions of spectral endmembers to the pixel spectrum.

Endmember spectra are defined by choosing representative pixels in the image and we refer to the spectrum of a pixel as its pseudo-spectrum since it is represented in DN (digital number) values (0-255) rather than radiance or reflectance values. Although the endmembers are at different elevations in the image, the choice of AVIRIS bands used in the model substantially reduces the influence of atmospheric effects on the model results. Only four of the 16 (or 18) bands used to model each image (Table 1) are in the visible wavelengths where the magnitude of atmospheric scattering effects depends on pixel elevation. One band is located in an atmospheric water vapor absorption region while the rest of the bands (10 or 11) span a spectral region where there is little atmospheric scattering and absorption. Thus elevation differences have a negligible effect on the model results.

Using Equation (2) we calculate the average root mean squared (RMS) error by squaring and summing the error term, $E_{\mathrm{c}}$ over the $M$ AVIRIS channels to show the fit of the model to the image data.

$$
\mathrm{RMS}=\left[M^{-1} \sum_{\mathrm{c}=1}^{M} E_{\mathrm{c}}^{2}\right]^{\frac{1}{2}}
$$

The RMS error is a combination of errors resulting from both the choice of endmembers and the error introduced by sensor noise. RMS values $\left(\mathrm{Wm}^{-2} \mu \mathrm{m}^{-1} \mathrm{sr}^{-1}\right)$ are transformed to $\mathrm{DN}$ values $(0$ 255) and displayed as an image so that spatial patterns of error can be investigated.

Two multi-band subset images, Tioga Pass-1989 and Mammoth Mountain-1991, were used in this analysis,

Table 1. AVRIS bands and corresponding band center wavelengths used for the spectral mixture model

\begin{tabular}{cccc} 
Band & Wavelength & Band & Wavelength \\
& $\mu \mathrm{m}$ & & $\mu \mathrm{m}$ \\
& & & \\
\hline & & & 1.03 \\
$7^{*}$ & 0.46 & 70 & 1.04 \\
16 & 0.55 & 71 & 1.05 \\
27 & 0.66 & 72 & 1.06 \\
38 & 0.72 & 73 & 1.07 \\
51 & 0.85 & 74 & 1.08 \\
$60^{*}$ & 0.94 & 75 & 1.09 \\
67 & 1.00 & 76 & 1.10 \\
68 & 1.01 & 77 & 1.11 \\
69 & 1.02 & 78 &
\end{tabular}

* Noisy bands in the 1989 data; not used for modeling the Tioga Pass image. containing 16 and 18 spectral bands in the visible and near-infrared wavelengths, respectively. These bands, shown in Table 1, were selected on the basis of spectral information content and signal-to-noise ratio in order to reduce redundancy and image noise and to facilitate image processing. Collected on 26 May 1989, the Tioga Pass scene covers an area of approximately $4 \times 4.4 \mathrm{~km}$ and contains patchy snow, thin snow, and deep snow areas. Vegetation in the scene is neither dense nor continuous with thin, rocky soil and senesced grasses and small shrubs visible in the areas where snow is absent. Tioga Lake was partially covered by a slushy ice layer but remained unfrozen around the edges. Concurrent with the AVIRIS overflight, snow reflectance and snow depth measurements were made and snow samples collected to characterize the effects of snow and grain size on spectral reflectance. The Mammoth Mountain image was acquired on 23 March 1991, the day after a major snowstorm deposited over $10 \mathrm{~cm}$ of fine-grained new snow over $2-3 \mathrm{~m}$ of existing snowpack. It covers an area of approximately $8 \times 6.9 \mathrm{~km}$. Unlike the Tioga Pass-1989 scene, this scene has snow-covered frozen lakes, deep snow in nearly all snow-covered areas, more densely forested areas, and more rugged topography producing substantial shading.

Unique endmembers were identified using 3-band color composites for each scene. Endmembers for the Tioga Pass 1989 image were Rock/Soil, Water, Vegetation, and Snow. For the Mammoth Mountain-1991 image, the endmembers chosen were Vegetation, Shade, and Snow. Because of the spectral similarity between lake water and shade (in the Tioga Pass 1989 image) and between dark rock and shade (in the Mammoth Mountain-1991 image) only one of the two for each was used as an endmember. The mixing model was run for each image to calculate the fraction of each endmember's spectral contribution to each pixel's pseudo-spectrum. Deviations between the model and the data were calculated as residuals and an RMS error image as well as an overall RMS error were computed.

\section{RESULTS}

Overall RMS error for the Tioga Pass 1989 scene was $3.4 \mathrm{Wm}^{-2} \mu \mathrm{m}^{-1} \mathrm{sr}^{-1}$ and for the Mammoth Mountain 1991 scene it was $3.0 \mathrm{Wm}^{-2} \mu \mathrm{m}^{-1} \mathrm{sr}^{-1}$, both of which are quite low. Displaying the RMS images allows the user to examine the spatial pattern of RMS error and to assess if additional endmembers are needed to model the data better. In the case of the Mammoth Mountain 1991 scene, highest RMS errors $\left(\approx 7 \mathrm{Wm}^{-2} \mu \mathrm{m}^{-1} \mathrm{sr}^{-1}\right)$ were associated with directly illuminated areas of snow in the upper basin areas on the south side of Mammoth Mountain. These basins have concave profiles and the light bouncing off adjacent slopes creates an additional source of variability that is not modeled. The spectral composition of this secondary illumination is different from that of the sun and, because of the complex topography, not all pixels are equally effected. The RMS image for Tioga Lake 1989 showed that highest errors $\left(6.25 \mathrm{Wm}^{-2} \mu \mathrm{m}^{-1} \mathrm{sr}^{-1}\right)$ are associated with the partially frozen slushy ice surface of Tioga Lake. 


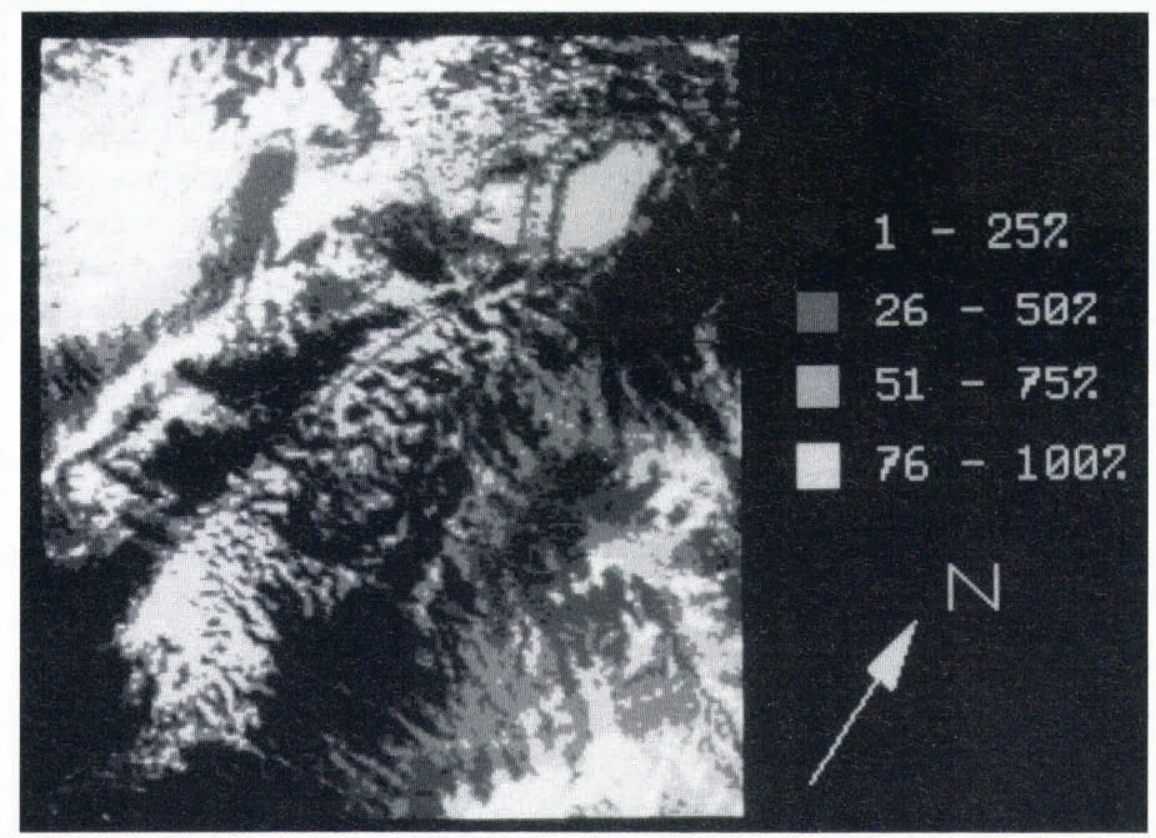

Fig. 1. This Tioga Pass image shows the fraction of snow cover in each pixel as calculated by the spectral mixture model. Tioga Lake is in the upper right region of the image and the road through Tioga Pass runs along the left side of the lake, from upper right to lower left. The image has been scaled so that black pixels represent a snow cover of $0 \%$, dark-gray pixels represent 1-25\% snow cover, medium-gray pixels represent 26-50\% snow cover, light-gray pixels represent 51$75 \%$ snow cover, and white pixels represent $76-100 \%$ snow cover. White pixels also appear to correspond to areas of deep snow while medium- and light-gray pixels may correspond to thin snow areas. This image, derived from an AVIRIS image sub-scene, represents an area of $4 \times .4 .4 \mathrm{~km}$.

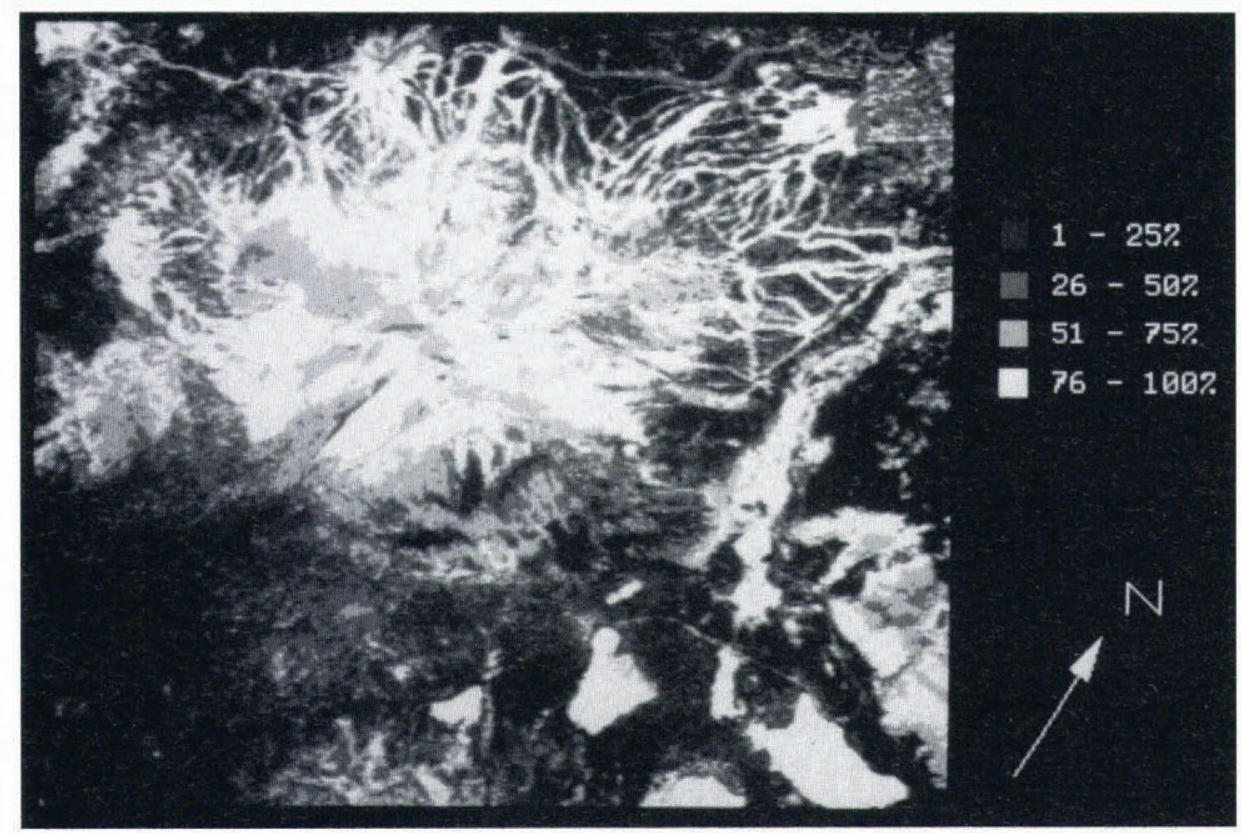

Fig. 2. This Mammoth Mountain snow cover fraction image shows the mixing model estimates of snow-covered area in each pixel. North is to the upper right of the image and the ski runs at the Mammoth Mountain ski resort are clearly seen in the upper portion of the image. This image, derived from an AVIRIS image sub-scene, represents an area of $8 \times 6.9 \mathrm{~km}$. The mountain ridge runs from upper left to the center of the image and several frozen, snow-covered lakes are visible in the lower right portion of the image. The gray scale is the same as in Figure 1.

Another means of examining the goodness of fit of the endmembers is to produce a fraction image of each endmember. Fractions are displayed in an 8-bit image with values of $0-100$ for fractions less than zero, values from $100-200$ for fractions ranging from $0-1$, and values from 200-255 for fractions greater than 1. Fraction overflow (fractions greater than 1) indicate that there were pixels in the image that were purer representations of that endmember. Fraction underflow (fractions less than 0 ) are those pixels that were not well represented by 
any of the endmembers. In all fraction images for both scenes, there was very little fraction overflow but some fraction underflow in the vegetation fraction images. This points to the difficulty in choosing vegetation endmembers in scenes where vegetation is discontinuous and not dense. However, since the goal was to map snow cover in both images, no further endmember manipulation was done since the snow fraction images showed only minor amounts of fraction over- and underflow.

Figures 1 and 2 show the snow fraction images for Tioga Pass 1989 and Mammoth Mountain 1991, respectively. In these fraction images, the snow fraction for each pixel is mapped on a gray scale with black $=0$ $5 \%$, dark gray $=6-25 \%$, medium gray $=26-50 \%$, light gray $=51-75 \%$, and white $=76-100 \%$.

\section{DISGUSSION AND CONGLUSIONS}

An important point to consider in the interpretation of the fraction images is that what we have actually calculated is the per cent contribution of the endmember pseudo-spectrum to the pixel pseudo-spectrum. What remains to be done is to relate this to the areal coverage of each endmember in each pixel. This relationship may depend on both snow thickness and the spatial arrangement of endmembers. The snow cover fraction image should still stand as a reasonable proxy image for fraction of snow-covered area.

The accuracy of the results is also influenced by the fact that the model is a linear mixing model and is being applied to a somewhat nonlinear problem. Nonlinearities are generated by the adjacency effect of bright pixels next to dark pixels adding some unaccounted-for path radiance'and by the anisotropic distribution of reflected radiation from snow (particularly in the near-infrared wavelengths), as well as by the presence of absorbing impurities in the snow (Tioga Pass 1989) that is best modeled as an intimate mixture (Johnson and others, 1983).

The interpretations of the snow cover fraction images for Tioga Pass 1989 and Mammoth Mountain 1991 scenes are somewhat different. Recall that the Tioga Pass 1989 scene contains areas of thin snow as well as patchy snow surrounded by vegetation and rock/soil. Concurrent reflectance measurements of thin snow over a dark substrate have shown that the spectral reflectance is a combination of both the snow reflectance, decreases with wavelength, and the substrate reflectance, which increases with wavelength (Nolin and Dozier, 1991). It is possible that, in areas of thin snow, the mixture model is calculating the fractions of endmembers which are superimposed (snow over rock/soil) rather than adjacently located.

In conclusion, using imaging spectrometer data and a spectral mixture modeling technique, we have successfully mapped deep snow, thin snow, shaded snow and snow mixed with vegetation. With careful interpretation, similar results could be used as input to hydrologic and climatologic models requiring estimates of snow-covered area.

\section{ACKNOWLEDGEMENTS}

We thank the Jet Propulsion Laboratory, Pasadena, California for providing AVIRIS data and Steve Willis of the University of Washington, Geological Sciences and Remote Sensing Laboratory for programming assistance with the WISP (Washington Image and Spectral Package) software. This work was supported by NASA grant NAGW 1265.

\section{REFERENCES}

Adams, J.B., M. O. Smith and P.E. Johnson. 1986. Spectral mixture modeling: a new analysis of rock and soil types at the Viking Lander 1 site. 7. Geophys. Res., 91(B8), 8098-8112.

Boardman, J. W. and A. F. H. Goetz. 1991. Sedimentary facies analysis using AVIRIS data: a geophysical inverse problem. In Green, R. O., ed. Proceedings of the Third Airborne Geoscience Workshop: AVIRIS. Pasadena, CA, Jet Propulsion Laboratory, 4-13.

Clark, R. N., G. A. Swayze, A. Gallagher, N. Gorelick and F. Kruse. 1991. Mapping with imaging spectrometer data using the complete band shape leastsquares algorithm simultaneously fit to multiple spectral features from multiple minerals. In Green, R. O., ed. Proceedings of the Third Airborne Geoscience Workshop: AVIRIS. Pasadena, CA, Jet Propulsion Laboratory, 2-3.

Dozier, J. 1989. Spectral signature of alpine snow cover from the Landsat Thematic Mapper. Remote Sensing Environ., 28, 9-22.

Dozier, J. and D. Marks. 1987. Snow mapping and classification from Landsat Thematic Mapper data. Ann. Glaciol., 9, 97-103.

Goetz, A. F. H., G. Vane, J. E. Solomon and B. N. Rock. 1985. Imaging spectrometry for Earth remote sensing. Science, 228, 1147-1153.

Johnson, P. E., M. O. Smith and J. B. Adams. 1983. A semiempirical method for analysis of the reflectance spectra of binary mineral mixtures. 7. Geophys. Res., 88(B4), 3557-3561.

Nolin, A. W. and J. Dozier. 1991. Spectral reflectance of thin snow. In Hunt, J.J., ed. Proceedings of the 5th International Colloquium. Physical Measurements and Signatures in Remote Sensing, Courchevel, France. Noorduijk, European Space Agency, 439-442. (SP-319.)

Roberts, D. A., M. O. Smith, J. B. Adams, D. E. Sabol, A. R. Gillespie and S. C. Willis. 1990. Isolating woody plant material and senescent vegetation from green vegetation in AVIRIS data. In Green, R.O., ed. Proceedings of the Second Airborne Geoscience Workshop: AVIRIS. Pasadena, CA, Jet Propulsion Laboratory, 42-57.

Sabol, D. E., J.B. Adams and M.O. Smith. 1992. Quantitative subpixel spectral detection of targets in multispectral images. 7. Geophys. Res., 97(E2), 2659 2672.

The accuracy of references in the text and in this list is the responsbility of the authors, to whom queries should be addressed. 